\title{
Computer Training in Cognitive Remediation of the Traumatic Head Injured
}

\author{
Dr. Jack Rattok, Executive Director \\ Transitions of long Island \\ LIJ Medical center \\ Manhasset, NY 11030
}

Traumatic head injury is distinct from other forms of disability in that the impact is direct and multiple upon the physical, emotional, and cognitive functioning of the disabled person. While therapies dealing with the physical and emotional aspects of the disability have been in existence for a relatively long period of time, cognitive remediation has not been available for victims of head trauma, and has remained mainly within the province of special educators working with the neurologically impaired child.

Research in cognitive remediation with stroke patients was begun at Rusk Institute, NYU Medical Center in the early 1970's and expanded into cognitive remediation with the traumatically head injured in the early 1980's. (Rattok, J., Ben-Yishay, Y., Thomas,J. L., Ross,B. 1981). Diller and Gordon (1981) in a summary article describing issues in cognitive remediation with neurologically impaired adults, indicate that there has been relatively little work done in the area.

Until 1984, the majority of cognitive training was done using paper and pencil tasks, and simple educational tools such as cubes and cards, which derived from special education techniques. Some researchers recognized the potential inherent in using computers for cognitive remediation, but they were expensive, used mainly for research in the area, and not in common use as training tools. In 1984, when the less expensive personal computers appeared on the market, training became computerized and is currently a common tool for cognitive remediation in rehabilitation facilities.

Computerized training has many technical advantages over the remedial tools which preceded. A variety of input devices: keyboard, joy stick, mouth, trackball, etc. can be selected to suit the needs of a disabled person. Visual devices (monitors) can be color or monochrome and appear in different sizes and shapes, enabling flexible adaptation to a patients visual needs. The computer is accurate, consistent, and stores a large amount of information in small space. Since it is a new and popular tool, it appeals to patients as an instrument of remediation.

Since cognitive training is not the purview of any single professional discipline, a variety of therapists have adapted these computerized tools to their use. While the physical and emotional aspects of head trauma are attended to by specific 
areas of medicine and psychology, cognitive remediation services that employ computer software are rendered by neuropsychologists, speech therapists, occupational therapists, special educators. Our survey showed that cognitive rehabilitation services are generally provided by: speech therapists (40\%); occupational therapists (30\%); neuropsychologists $(10 \%)$; cognitive technicians (20\%). Cognitive technicians represent a newer form of service. They are individuals trained in the implementation of specific exercises (mainly through use of the computer) and their work is a technical follow-up and reinforcement of the domains dealt with by the other disciplines cited. However, there remains question as to whether the currently used computer software proves effective in remediating the cognitive-intellectual malfunctions experienced by traumatically head injured patients.

The software that is currently available covers four major domains of cognitive remediation: (1) eye-hand coordination; (2) basic attention; (3) basic academic skills; (4) problem solving.

Computerized eye-hand coordination tasks are games such as "Pac-Man" and "Space Invaders." Basic attention tasks are generally made up of packaged software developed out of cognitive psychology (Ben-Yishay, Piasetsky, and Rattok, J. 1987. Basic academic skills training consists mainly of software developed for schools by special educators and the majority contain drill exercises in reading comprehension and arithmetic. Problem solving techniques are usually based on material designed to advance the problem solving capability of school age children. In sum, currently available software is geared mainly toward augmenting basic cognitive skills.

Most publications available concerning software for cognitive remediation consist of non-critical lists of the software available and their sources (Kreutzer, Hill, and Morrison, 1987). Some publications review software and give the reviewer's impressions, but have no base in research (Lynch, 1985.) Others dissect software technically and provide a thorough description of the software, but have no theoretical orientation and do not present outcome measures. A few papers dealing with training outcomes have been published, (Kerner, and Acker,1985). These papers are not comprehensive nor numerous enough to allow any conclusions about the efficacy of computer software for cognitive retraining.

In recent years more and more publications start to appear, researching and organizing the various elements that are necessary to produce comprehensive products to remediate various aspects of cognitive deficits (Rattok 1992, Rattok and Ross 1992, Kaufman and Rattok 1992).

As personal computers became widely available in the mid-1980's, it became evident that they could serve to replace these costly and commercially unavailable electronic devices, and had important applicability as a more accurate and efficient tool to replace the use of paper and pencil tasks. Objections that "the 
computer will replace the clinician" in the decision-making process were soon dispelled. Professionals began to use computers and came to understand them as an elaborate tool, rather than an intellectual entity.

Recent findings indicate that for some psychological testing the patient tends to be more honest and provides the right information to the computer screen then to the clinician. (Fillon, 1990)

The main advantage of computerized psychological testing with the Head Injured involved the Visual perceptual difficulties experienced by this disabled population. Most common inventories (MMPI, SCL, CPI) usually cram hundreds of questions onto a few pages and employ computerized answer sheets that confuse and overload patients with visual and perceptual demands. In order to maximally control for perceptual malfunctions we computerized the tests so that each question appears clearly by itself on the screen together with the answer choices. The computer automatically records both the answer and the time that it takes to answer each question. This arrangement yields the ability to score performance more accurately than the usual pen and pencil administration.

Example (Rattok, \& Ross, 1993): The PENN Inventory for Post Traumatic Stress Disorder (PTSD) was administered to Head Injured subjects. It consists of 26 sets of statements. In each set, the subject must choose the statement that best describes his situation. In order to maximally control for perceptual malfunction we computerized the tests so that each set of questions appears clearly by itself on the screen. The computer automatically records both the answer and the time that it takes to answer each question. This arrangement is more suitable for the head injured with various cognitive disorders (Rattok \& Ross, 1986), and also yields the ability to score performance more quickly and accurately than the usual pen and pencil administration. All subjects were tested in individual sessions in order to assess their functioning on the PENN Inventory.

The paper and pencil version displayed 10 sets of questions per page and was so confusing that most of the subjects in the experiment refused to participate.

Fig 1. Exemplified the way the questions were presented on the Computer screen.

The outcome is presented on the secern immediately (Fig. 2) and includes normative comparison to other population. Similar capacities make the actual cognitive training exercises more successful then their pencil and paper counter part.

Visual exercises that involve complex pictorial presentations can not be part of a training program without adequate software. An example for such an exercise can be seen in Fig. 3. The picture on the screen is the normal picture, the picture in Fig. 4 was scrambled and the patient has to rebuild it (the original screen is in $640 \times 480$ resolution 256 colors). 
QUESTION - 1

1) I don't feel much different than most other people my age.

2) I feel some what different than most people my age -

3) I feel so different than most other people my age that I choose pretty carefully who I' 11 be with and when.

4) I feel so totally alien to most other people my age that I stay away from all of them at all costs.

Fig. 1 Item screen (PTSD)

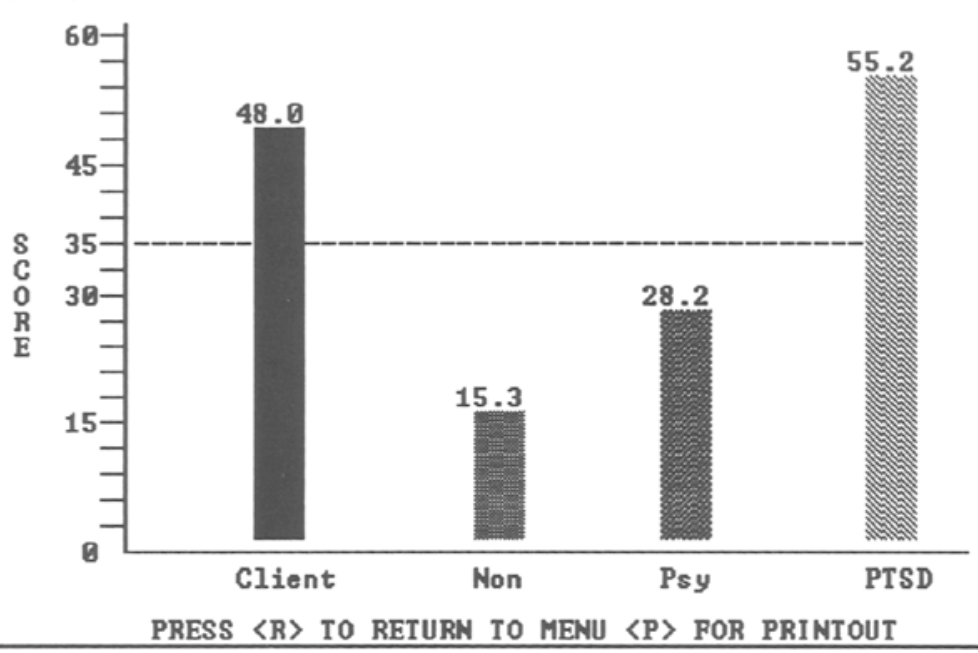

Fig. 2 Score screen (PTSD) 


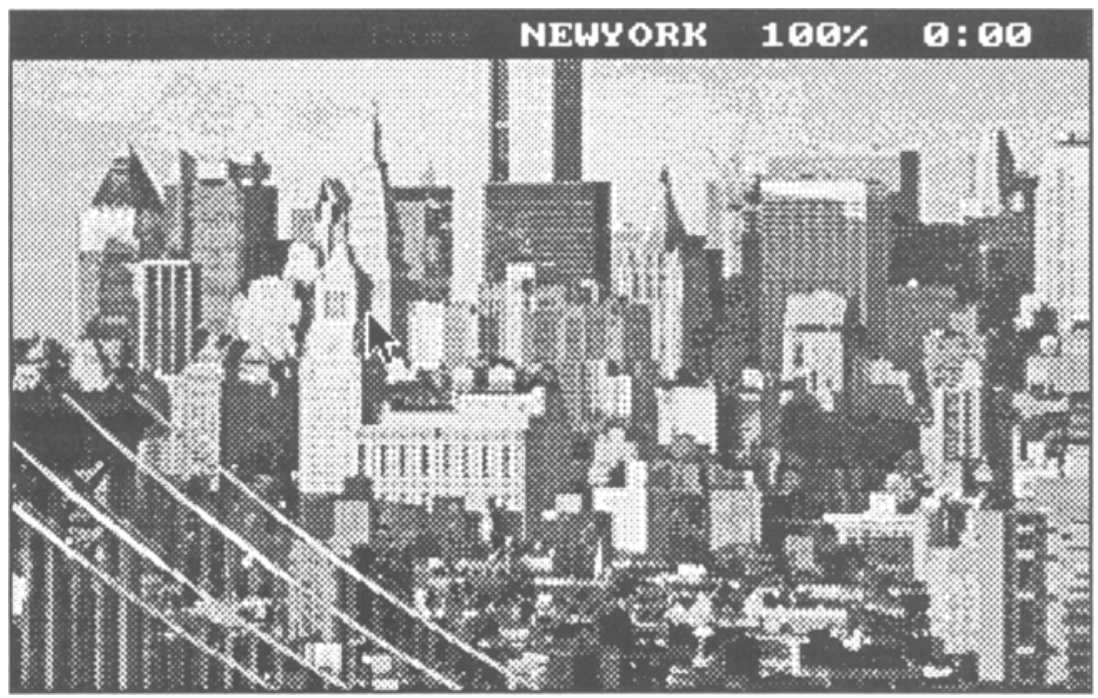

Fig. 3 New York skyline unscrambled

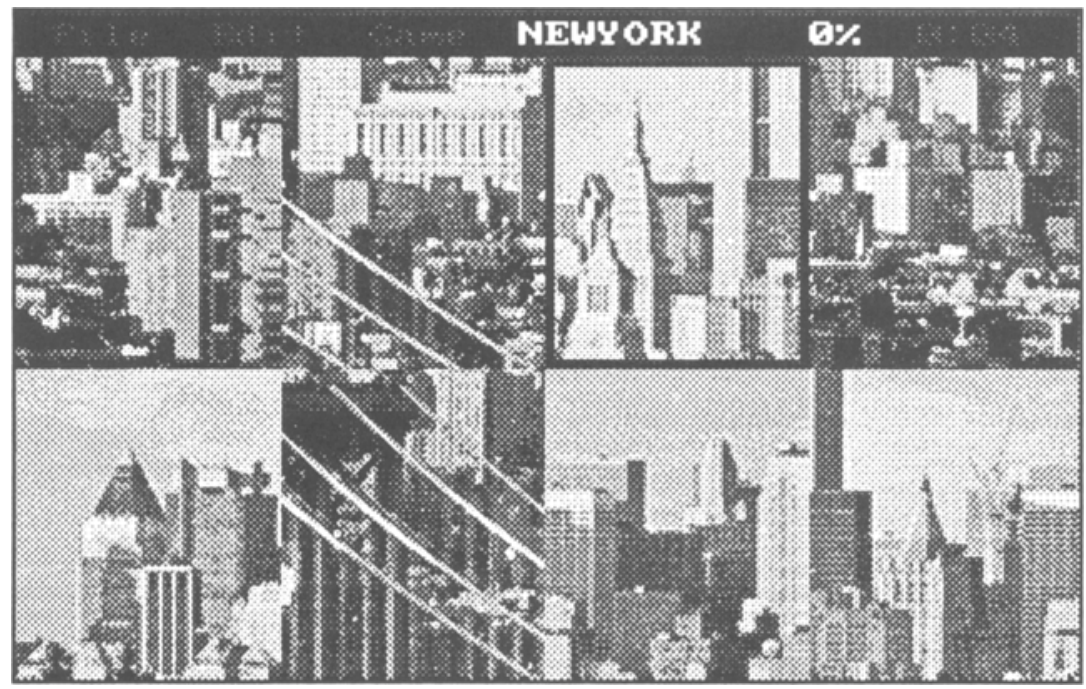

Fig. 4 New York skyline scrambled 


\section{Hardware Consideration}

Numerous amounts of special hardware for the physically disabled is available, however a big part of this hardware is not useful for the head injured since in addition to the physical disability they acquire cognitive (intellectual and severe memory deficits) disability and thus can not learn how to use the sophisticated systems available for them.

In the early 1980 's most software for cognitive retraining was written for the Apple II line of computers. With the reduction of cost most clinical settings moved to use IBM and IBM compatible (MS DOS) computers. At the time the Apple line moved to the Macintosh model. The majority of the software is written by professional providers that come mainly from the medical and paramedical fields (not computer specialists). Most of this software written in the late 80's into the nineties is written for the Intel Microsoft combination (MS DOS) and it is hard currently to find a choice of software for cognitive retraining operable on the Apple Macintosh line. Our discussion on hardware choice will center on the IBM and IBM compatible computer.

\section{CPU.}

The current trend in the software for cognitive retraining shows a move toward using the Windows environment for the new programs coming out. Thus choice of CPU today has to conform with adequate hardware that will support this environment. Currently it means at least $486 \mathrm{SX}$ processor running on $25 \mathrm{Mhz}$ with 4 Mbytes of memory, 170 Mbytes hard disk drive and a SVGA capable of running $640 \times 480$ with 256 color monitor.

We would like to indicate that some providers keep a few old 8086 computers since early software was written without use of timed delays and work correctly on the old 8086 CGA computers, but run too fast on the 386 and 486 machines. Check the software that you buy and don't buy it if you do not have one of the old machines available.

\section{Monitors.}

Patients suffering from head injury usually have multiple visual problems and thus need a screen presentation that is relatively large. However, the majority of them also have visual scanning disorders (spatial neglect) and thus will omit some information presented on a big monitor. If the clinic has more than one system for cognitive retraining buy various sizes; 12", 14", 15", and 17" will be most adequate. If only one system is available the popular $14^{\prime \prime}$ will do. 


\section{Input Devices.}

Most head injured patients experience (at least in the first year post their injury) motor control and coordination difficulties, thus the use of regular keyboards is inadequate. Special keyboards for the disabled are available to compensate for this problem. For our clinic, we found most versatile the Intelkey keyboard manufactured by Unicorn Engineering. Usage of non keyboard input devices like a mouse stylus etc, can be problematic. The input device that we found to work well is a trackball (the bigger the better) since in using a trackball it is enough to be able to coordinate two to three fingers rather then the whole hand (as is necessary with a mouse).

\section{Audio Devices.}

Speech therapist working with the head injured are using successfully microcomputer equipped with an audio board to improve communication skills of their clients. The majority of the software was written geared toward propriety, or the Covox standard. The general standard that govern the audio boards in the MSDOS market today, is the AdLib Sound Blaster standard some boards are capable of handling multiple standards. Unless a therapist is using only one specific software package, make sure that the audio board installed in the cognitive retraining system is a multi standard board.

\section{Printers.}

The printer seems to be the least problematic part of the system. We noticed that with the lack of normal eye hand control in these patients, printers using continuous feed paper are hard to handle. If you want your patient to be as independent as possible use a cut sheet feeder (most inkjet printers are capable of handling regular noncontinuous printer paper and are a good choice for cognitive retraining systems.

\section{Multimedia Devices}

The capacity of multimedia devices seems to be very promising and probably will augment the quality of cognitive training. Unfortunately current scientific literature of cognitive rehabilitation does not address this topic. Therapist who would like to experiment with multimedia systems should install MPC level 2 systems. Such system will include double speed CD-ROM, 16 bit sound card, and speakers. 


\section{References}

Ben-Yishay, Y., Piasetsky, E. B., and Rattok, J. (1987). A Systematic Method for Ameliorating Disorders in Basic Attention. In Neuropsychological Rehabilitation, Meir, M. J., Diller, L., and Benton, A., L. (Eds.). Livingstone, London, p. 165-181.

Diller, L., and Gordon, W.A. (1981). Intervention for cognitive deficits in brain injured adults. Journal of consulting and clin. Psychology. 49, 822-834.

Fillon, M. Face to Face or Interface. Information Week, 281, New York, 1990.

Kaufman B., Rattok J. A Computerized Training program to Facilitate Word Retrieval. A paper presented in: Technology and Person with Disabilities. University of California, Northbridge, 1992

Kerner, M.J., and Acker, M. (1985). Copmputer Delivery of Memory retraining with Head Injured Patients. Cognitive Rehabilitation. November, 26-31.

Kreutzer, J.S., Hill, M.R., and Morrison, C. (1987). Cognitive Rehabilitation Resources for the Apple II Computer. NeuroSience Publishers, Indianapolis.

Lynch, B. (1985). Captain: Cognitive Training Series. Software review. Cognitive Rehabilitation. November, 32-34.

Rattok, J., and Ross, B. The Use of Personality Inventories with the Traumatically Head Injured. Journal of Clinical and Experimental Neuropsychology, Vol. 10, January 1987.

Rattok J. Computer assessment of three dimensional visualization. A paper presented in: Technology and Person with Disabilities. University of California, Northbridge, 1992.

Rattok J., Ross B.: A Practical Approach to Cognitive Rehabilitation. NeuroRehabilitation, An Interdisciplinary Journal, Vol. 2, No. 3, 1992.

Rattok J., Ross B. Post Traumatic Stress Disorder (PTSD) in the Traumatically Head Injured (THI). Journal of Clinical and Experimental Neuropsychology, Vol. 16, March 1993.

Rattok J., Ross B. Cognitive Rehabilitation. In: Silver, Yudofsky, \& Hales (Eds.) Psychiatric Aspects of Traumatic Brain Injury. American Psychiatric Press, Washington D.C, 1994. 